\title{
Prevención secundaria post infarto agudo de miocardio en hospitales públicos: implementación y resultados de las garantías GES
}

\author{
CAROLINA NAZZAL ${ }^{1, a}$, FERNANDO LANAS ${ }^{2}$, MARÍA LUISA GARMENDIA ${ }^{3}$, \\ CLAUDIO BUGUEÑO ${ }^{4}$, ENRIQUE MERCADAL ${ }^{5}$, EDUARDO GARCÉS ${ }^{6}$, \\ PATRICIO YOVANINIZ7 ${ }^{7}$ PATRICIO SANHUEZA ${ }^{8}$
}

\section{Universal health coverage and accomplishment of secondary prevention goals among patients with acute myocardial infarction}

Background: In 2005, acute myocardial infarction (AMI) was included in a universal health plan (GES) to reduce inequity in care and optimize its diagnosis and treatment. Aim: To evaluate the effect of GES in risk factor control and therapeutic management among patients with AMI. Material and Methods: A survey was conducted in 2008-2009 in six public hospitals. Patients were identified from a hospital based registry of AMI and evaluated one year later with laboratory tests and an interview. Results: The registry enrolled 534 patients with ST and non ST segment elevation myocardial infarction. Of these, 416 patients aged $63 \pm 12$ years (25\% women) were evaluated one year later. Eighty three percent were evaluated by a cardiologist and $37 \%$ by a general practitioner. Twenty two percent were evaluated by a nurse and $22 \%$ by a nutritionist. At the moment of the interview, $9 \%$ smoked, $78 \%$ were overweight or obese, $24 \%$ performed moderate or vigorous physical activity $\geq 150 \mathrm{~min} /$ week, $60 \%$ had systolic pressure $>130 \mathrm{mmHg}$ and $63 \%$ a diastolic pressure $>80 \mathrm{mmHg}$. In 30\%, LDL cholesterol was $>100 \mathrm{mg} / \mathrm{dl}$ and in 43\%, triglycerides were $>150 \mathrm{~mm} / \mathrm{dl}$. Twenty two percent were diabetic and among them, $52 \%$ had a glycosilated hemoglobin $>7 \%$. Forty five percent of non-diabetic patients had a fasting glucose $>100 \mathrm{mg} / \mathrm{dl}$. Ninety three percent were in treatment with aspirin, 86\% with statins, $66 \%$ with $\beta$-blockers, and $73 \%$ with angiotensin converting enzyme inhibitors or angiotensin receptor blockers and 20\% with clopidogrel. Conclusions: Despite the high proportion of patients in treatment with evidence-based therapy, many do not achieve the targets for risk factor control with the new health care model.

(Rev Med Chile 2013; 141: 977-986).

Key words: Anterior wall myocardial infarction; Chile; Practice guideline; Quality of health care; Secondary prevention; Universal coverage.

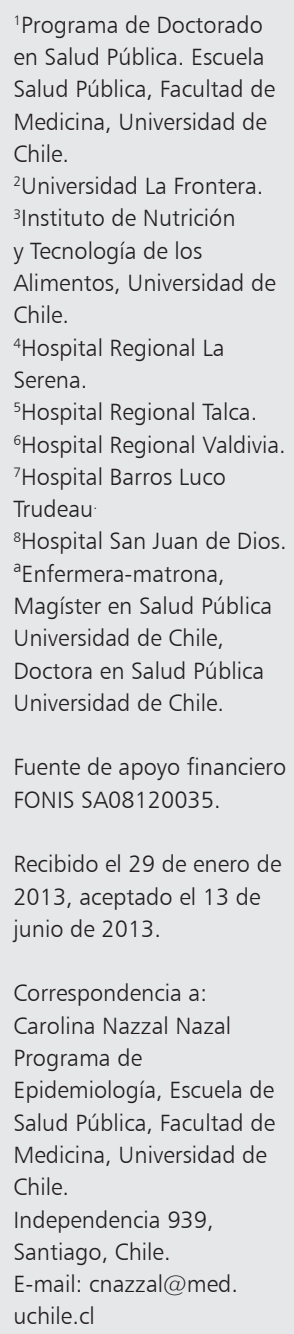

E n Chile la enfermedad coronaria es la segunda causa de muerte en ambos sexos, constituyendo un problema de salud pública ${ }^{1-3}$. Dado que los factores de riesgo cardiovasculares se asocian a la patogénesis y a la progresión de la enfermedad, su control y tratamiento mejoran el pronóstico ${ }^{4-10}$.
Numerosa evidencia muestra que las intervenciones en los estilos de vida ${ }^{11-15}$ y el control de los factores de riesgo aumentan la sobrevida y la calidad de vida en pacientes coronarios ${ }^{16-22}$, justificando la necesidad de contar con programas de prevención secundaria que aborden estos aspectos ${ }^{12,23-25}$. 
En 2005 en Chile se dio inicio al Régimen de Garantías Explicitas en Salud (GES), cuyo objetivo fue mejorar la atención de salud de la población y corregir las inequidades en salud. El infarto agudo de miocardio (IAM) fue incluido en el primer grupo de 56 patologías incorporadas a este nuevo modelo de atención, mediante el cual el Estado resguarda el acceso, la oportunidad, la cobertura financiera y la calidad de la atención médica de estas patologías ${ }^{26}$. Para ello se estableció una guía clínica con recomendaciones abarcando desde el diagnóstico hasta la prevención secundaria ${ }^{27}$, y se definió su implementación en el nivel primario y secundario ${ }^{28}$. Una primera evaluación mostró reducción en la letalidad intrahospitalaria del IAM posterior a la puesta en marcha de las GES, asociada a mejoría en la terapia y a la mayor utilización de procedimientos de reperfusión y revascularización miocárdica ${ }^{29}$.

Los objetivos del presente trabajo son i) determinar el grado de control de los factores de riesgo al año post-IAM y ii) evaluar el cumplimiento de las recomendaciones del programa de prevención secundaria post infarto con posterioridad a la puesta en marcha de GES.

\section{Materiales y Métodos}

\section{Población y diseño}

Pacientes con IAM con y sin supradesnivel del segmento-ST (SDST) que hubiesen egresado vivos de una hospitalización por un primer episodio de infarto o infarto recurrente en seis hospitales públicos del registro hospitalario de infarto, Registro GEMI, y que fueron evaluados al año post-infarto. Se seleccionaron dos hospitales de Santiago y cuatro de regiones, todos centros de referencia y hospitales de alta complejidad, con unidad coronaria y laboratorio de hemodinamia, salvo en dos de ellos. El Registro GEMI es un registro hospitalario -público y privado- de pacientes que ingresan con diagnóstico de IAM. Los criterios de inclusión al registro son la presencia de elevación de enzima CK y su fracción MB al doble o más del valor normal o elevación de troponinas por encima del valor normal y, al menos uno de los siguientes: 1) dolor anginoso típico de al menos 30 min de duración; 2) alteración electrocardiográfica característica de $\mathrm{IAM}^{30}$. Este registro es representativo en cuanto a edad, sexo y previsión del universo de casos de
IAM, sin embargo, presenta una letalidad hospitalaria menor, posiblemente porque la mayoría de los centros que participan son hospitales de alta complejidad. Además los pacientes que fallecen precozmente no ingresan a las unidades coronarias y, por tanto, tampoco al registro GEMI.

En cada hospital fueron incluidos los pacientes que hubiesen ingresado entre julio de $2008 \mathrm{y}$ diciembre de 2008, identificados retrospectivamente del Registro GEMI. En tres centros se extendió el reclutamiento para alcanzar el tamaño de muestra estimado (Tabla1). Para la realización del control al año, los pacientes fueron contactados por teléfono o visitas domiciliarias y controlados en sus respectivos hospitales; se excluyeron aquellos sujetos con alteraciones cognitivas o de movilidad y quienes cambiaron de lugar de residencia.

\section{Información y manejo de datos}

Del Registro GEMI se obtuvo la información sobre antecedentes cardiovasculares, factores de riesgo, características del infarto, tratamiento farmacológico intrahospitalario, tratamiento al alta y evolución intrahospitalaria. El protocolo del estudio consideró un control por cardiólogo y enfermera al año post-infarto. Los pacientes fueron citados y examinados utilizando determinaciones estandarizadas que incluyeron examen físico, pruebas bioquímicas, y aplicación de un cuestionario. Se evaluó presión arterial (con esfigmomanómetro de mercurio), frecuencia cardiaca, capacidad funcional (según clasificación NYHA), peso (mediante balanza mecánica calibrada), talla, circunferencia cintura, perfil lipídico, glicemia, $\mathrm{HbAcl}$ en diabéticos, actividad física (mediante cuestionario IPAQ abreviado), tabaquismo (por autoreporte de número de cigarrillos/día promedio última semana); las pruebas bioquímicas se tomaron en ayuno y fueron procesadas en los laboratorios locales. Se preguntó por el lugar y número de controles durante el primer año, satisfacción usuaria (satisfacción respecto de profesionales de la salud en relación a tratamiento, trato personal y educación recibida, escala de 1 a 7) y características socio demográficas. Se registró la incidencia de eventos fatales y no fatales (nuevo infarto, angina inestable y revascularización miocárdica). En los pacientes fallecidos se revisaron las historias clínicas y se solicitó al Registro Civil el certificado y causa de muerte; en los pacientes no contactados se evaluó su estado vital al momento del cierre 
del estudio y en caso de muerte se obtuvo la causa del certificado de defunción. La información fue revisada verificando la calidad y completitud de los datos.

\section{Tamaño de muestra}

Se calculó que 384 sujetos controlados al año permitirían estimar una prevalencia de $50 \%$ de presión arterial elevada ${ }^{31}$, con error alfa de $5 \%$ y precisión de $5 \%$; se incluyó $20 \%$ más participantes por posibles pérdidas de seguimiento.

\section{Análisis estadístico}

Se realizó análisis exploratorio; en las variables continuas se evalúo normalidad con test de Shapiro-Wilk. Las variables categóricas se describieron mediante frecuencias absolutas y relativas y las continuas con cuartiles o media y desviación estándar. Se construyó para cada factor de riesgo una variable dicotómica utilizando los puntos de corte establecidos en la guía GES para prevención secundaria de IAM: colesterol total $<200 \mathrm{mg} / \mathrm{dl}$, LDL $<100 \mathrm{mg} / \mathrm{dl}$, triglicéridos $<150 \mathrm{mg} / \mathrm{dl}$; presión arterial sistólica $<130 \mathrm{~mm} /$ Hg y diastólica $<80 \mathrm{~mm} / \mathrm{Hg}$; glicemia $<100 \mathrm{mg} /$ dl; $\mathrm{HbAcl}<7 \%$ en diabéticos; IMC $<25 \mathrm{~kg} / \mathrm{m}^{2}$, ausencia de hábito tabáquico y actividad física moderada o intensa $\geq 150 \mathrm{~min} / \mathrm{semana}^{28,32,33}$ y se calculó el porcentaje de pacientes que cumplieron las metas. Se estimó la proporción de pacientes en tratamiento (en ausencia de contraindicaciones) con aspirina, betabloqueadores, inhibidores de enzima convertidora (IECA) o antagonistas de los receptores de angiotensina II (ARA II), estatinas y clopidogrel y se comparó con el tratamiento indicado al alta hospitalaria mediante prueba de $\chi^{2}$ para muestras pareadas. Se consideró significativo un valor $\mathrm{p}<0,05$ a dos colas, se empleó el software STATA 11.2 para los análisis (StataCorp. Texas, Estados Unidos).

\section{Ética}

El estudio contó con la aprobación del Comité de Ética de la Facultad de Medicina de la Universidad de Chile, al cual adscribieron los hospitales de la Región Metropolitana y de la IV Región, y de los Comités de Ética de los Servicios de Salud del Maule, Valdivia y Araucanía Sur. Todos los pacientes firmaron consentimiento informado para su participación.

\section{Resultados}

De un total de 643 pacientes que fueron ingresados por IAM en los seis centros y que no fallecieron durante la hospitalización, 534 (83,0\%) fue ingresado al Registro GEMI. La edad media fue $62,3 \pm 12,3$ años y la proporción de mujeres $24,7 \%$. De los pacientes elegibles $(n=493)$, en 416 $(84,4 \%)$ se obtuvo información al año y 36 (6,7\%) habían fallecido antes de este control (Figura1). Los sujetos en quienes no se realizó el seguimiento eran levemente más jóvenes y presentaron mayor proporción de IAM con SDST (Tabla 2).

En los pacientes evaluados al año, la edad media fue $61,9 \pm 11,9$ años y la proporción de mujeres 23,4\%. La mayoría estaba casado (70\%), había

Tabla 1. Número y proporción de pacientes con IAM incluidos en cada centro en las distintas etapas del estudio

\begin{tabular}{|lcccccc|}
\hline Hospital & $\begin{array}{c}\text { (A) Egresos } \\
\text { vivos } \\
\text { n }\end{array}$ & \multicolumn{2}{c}{ (B) Registro GEMI } & \multicolumn{3}{c|}{ Control al año } \\
\hline San Juan de Dios & 92 & $\mathbf{n}$ & \% (A) & $\mathbf{n}$ & \% (B) & \%(A) \\
\hline Barros Luco T & 116 & 63 & 68,5 & 41 & 65,1 & 44,6 \\
La Serena & 108 & 93,1 & 69 & 63,9 & 59,5 \\
\hline Talca & 140 & 121 & 86,4 & 104 & 86,0 & 74,3 \\
\hline Temuco & 101 & 94 & 93,1 & 83 & 88,3 & 82,2 \\
Valdivia & 89 & 61 & 68,5 & 45 & 73,8 & 50,6 \\
\hline Total & 105 & 87 & 82,9 & 74 & 85,1 & 70,5 \\
\hline
\end{tabular}




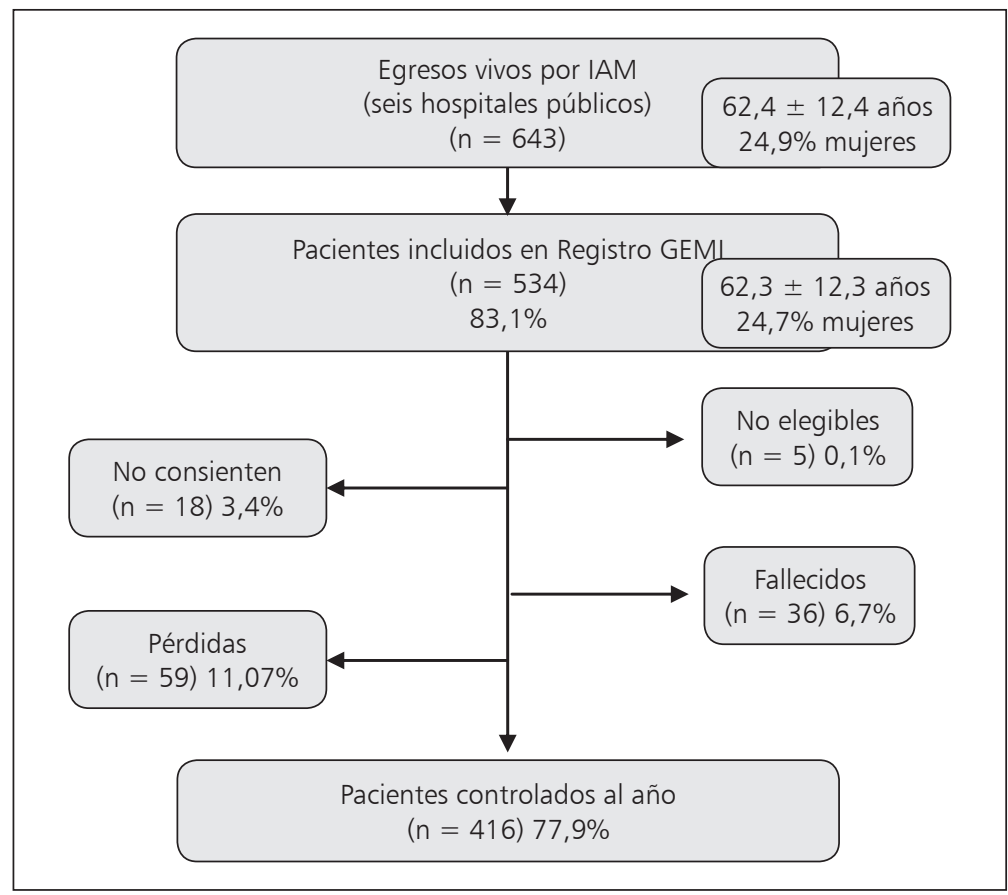

Figura 1. Configuración de la muestra.

Tabla 2. Comparación de características demográficas y clínicas basales entre pacientes con y sin seguimiento a un año

\begin{tabular}{|c|c|c|}
\hline Característica & $\begin{array}{l}\text { Pacientes controlados } \\
\qquad(n=416)\end{array}$ & $\begin{array}{l}\text { Pacientes no controlados } \\
\qquad(\mathbf{n}=\mathbf{8 2})\end{array}$ \\
\hline Edad, años (promedio $\pm \mathrm{DE}$ ) & $61,9 \pm 11,9$ & $59,1 \pm 10,7^{*}$ \\
\hline Sexo femenino (\%) & 23,4 & 17,5 \\
\hline Hipertensión arterial (\%) & 62,3 & 56,3 \\
\hline Diabetes (\%) & 22,2 & 16,2 \\
\hline Obesidad (\%) & 22,4 & 23,4 \\
\hline Colesterol, mg/dl (promedio \pm DE) & $183 \pm 52$ & $192 \pm 41$ \\
\hline Triglicéridos, mg/dl (promedio $\pm D E)$ & $151 \pm 107$ & $156 \pm 85$ \\
\hline Tabaquismo actual (\%) & 33,7 & 42,5 \\
\hline IAM previo (\%) & 8,3 & 7,5 \\
\hline IAM con SDST (\%) & 70,2 & $83,7^{*}$ \\
\hline
\end{tabular}

${ }^{*} p<0,05$.

cursado estudios básicos (50\%) y pertenecían a un nivel socioeconómico bajo: $80 \%$ percibía un ingreso familiar mensual menor a 300.000 pesos y $74 \%$ estaba afiliado a FONASA A o B. Los hombres eran significativamente menores que las mujeres $(60,3 \pm 11,4$ vs $67,0 \pm 12,1$ años; $\mathrm{p}<0,0001)$.

Características de la atención de salud: $60 \%$ tuvo 3 controles/año (p25-75:2-5) y la percepción acerca del personal de salud (médico, enfermera y nutricionista) fue excelente (mediana $=7$ ). En relación a los profesionales que habían participado durante el primer año en el seguimiento garantizado en GES, 85\% de los pacientes había sido controlado al menos una vez por cardiólogo 
y $38 \%$ por médico no especialista en atención primaria; en cambio la participación de enfermera y nutricionista sólo alcanzó a 21,5\% y 22,0\% respectivamente. Setenta y ocho por ciento refirió recibir los medicamentos regularmente.

\section{Factores de riesgo}

En la Tabla 3 se muestra la distribución de los factores de riesgo en el control al año. En relación al logro de las metas terapéuticas para los factores de riesgo evaluados (Figura 2), el menor porcen- taje de logro se encontró para el IMC $(22,0 \%)$ y para actividad física $(23,9 \%)$; con respecto a esta última nadie refirió participar de un programa regular de ejercicios. La prevalencia de sobrepeso y obesidad fue $44,8 \%$ y $33,2 \%$ respectivamente y no hubo variación en el peso promedio basal (al momento del infarto) con el obtenido al año (76,6 $\pm 14,3$ kilos vs $76,6 \pm 14,1$ kilos; $p=n s ;$ resultados en 292 pacientes). Setenta y cuatro por ciento de los pacientes dejó de fumar después del IAM; la prevalencia de tabaquismo al año fue $9 \%$ y la

Tabla 3. Distribución de la presión arterial, marcadores bioquímicos y medidas antropométricas al año post IAM

\begin{tabular}{|lccc|}
\hline Variable & n & p50 & (p25-p75) \\
Presión arterial sistólica (mmHg) & 411 & 130 & $120-150$ \\
Presión arterial diastólica (mmHg) & 411 & 80 & $70-90$ \\
Colesterol total (mg/dl) & 396 & 157 & $131-191$ \\
Colesterol LDL (mg/dl) & 390 & 83 & $61-107$ \\
Colesterol HDL (mg/dl) & 390 & 41 & $35-50$ \\
Triglicéridos (mg/dl) & 393 & 137 & $97-187$ \\
Glicemia (mg/dl) en no diabéticos & 299 & 98 & $90-107$ \\
Hemoglobina Ac1 en diabéticos (\%) & 78 & 7,0 & $6,2-9,0$ \\
Índice de masa corporal (kg/m²) & 406 & 28,4 & $25,2-31,2$ \\
Circunferencia cintura hombres (cm) & 305 & 98 & $92-105$ \\
Circunferencia cintura mujeres (cm) & 100 & 96 & $90-105$ \\
\hline Ejercicio (min/semana) & $410^{*}$ & 0 & $0-100$ \\
\hline
\end{tabular}

* En tres sujetos no se pudo evaluar por presentar limitaciones físicas severas para realizar ejercicio

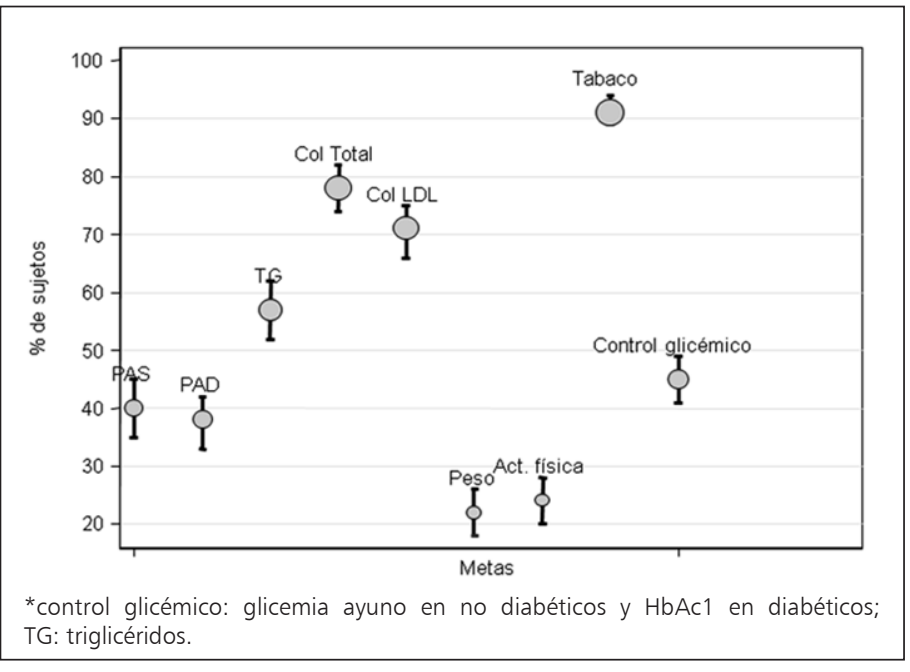

Figura 2. Porcentaje de logro para cada meta terapéutica de los factores de riesgo evaluados a un año post-infarto. 

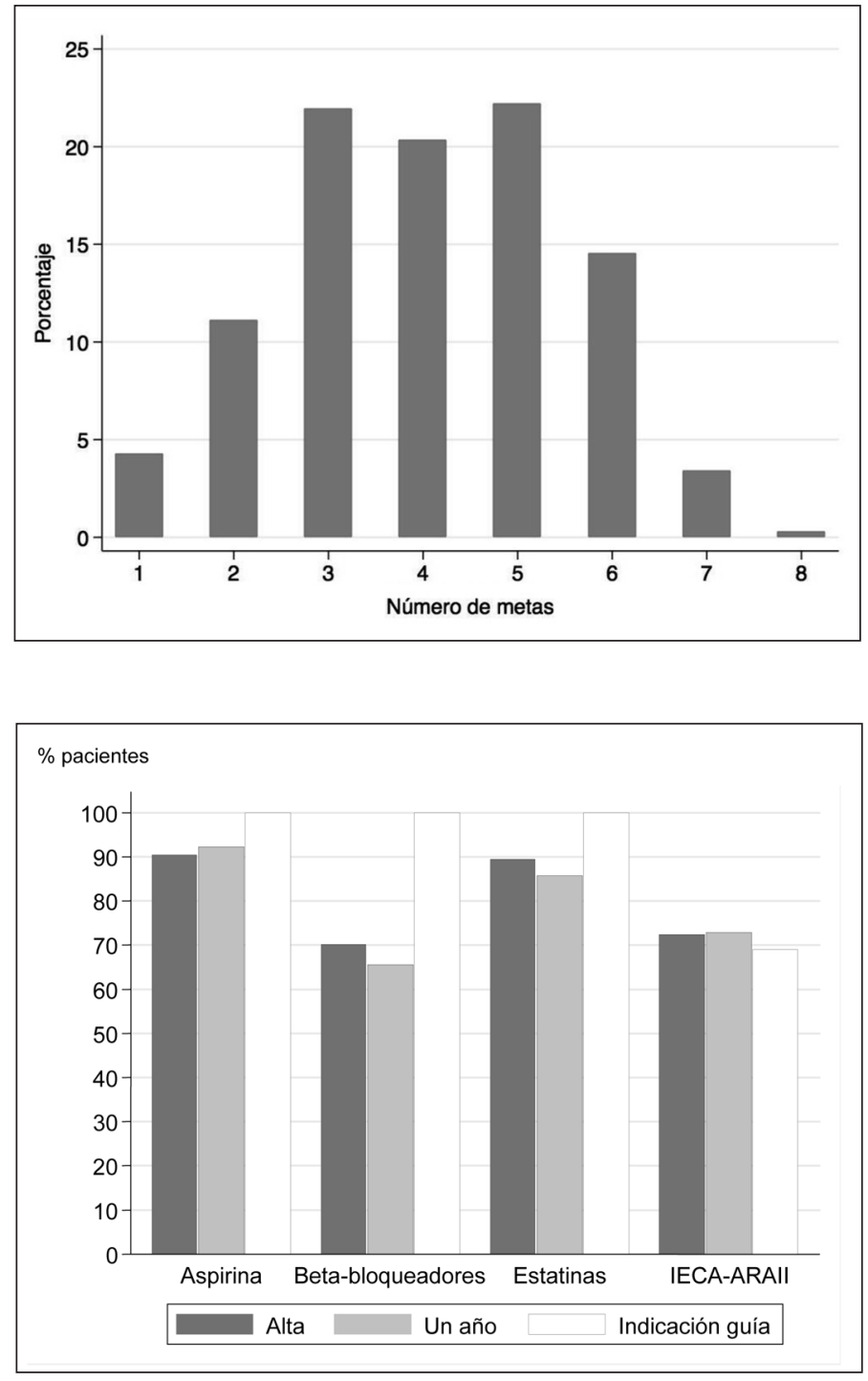

Figura 3. Tratamiento farmacológico al alta hospitalaria y al año post-infarto.
Figura 4. Número de metas cumplidas conjuntas para los factores de riesgo cardiovasculares al año post-infarto. mediana de consumo en ellos fue 6 cigarrillos/ día (p25-p75:3-14). En 351 casos se obtuvo información sobre las 8 metas evaluadas y $50 \%$ de los pacientes alcanzó 4 de ellas (Figura 3).

\section{Tratamiento y evolución}

La mayoría de los pacientes $(61,3 \%)$ se encontraba asintomático al momento del control, $16,5 \%$ había presentado angina de esfuerzo, $4,1 \%$ angina de reposo y $29,6 \%$ había evolucionado con insuficiencia cardiaca $(77,1 \%$ capacidad funcional II y $17,2 \%$ en capacidad funcional III).

En la Figura 4 se muestra el empleo de los fár- macos al alta y al año. A pesar que el porcentaje de contraindicaciones fue bajo (aspirina 3,6\%; betabloqueadores 7,7\%; IECA o ARAII 11,9\% y estatinas $1,9 \%$ ), no todos los pacientes que presentaban indicación se encontraban en tratamiento. De los pacientes tratados con angioplastía primaria, 33\% recibía clopidrogrel al año. No se encontraron diferencias en el tratamiento farmacológico según sexo (resultados no se muestran). En los pacientes de mayor edad la chance de recibir estatinas (OR $0,97 ; 0,94-1,00)$ y aspirina (OR 0,96; $0,94-1,00)$ fue levemente menor. Durante el primer año 21,1\% requirió una nueva hospitalización por causa 
cardiovascular (5,1\% isquemia recurrente; $2,4 \%$ nuevo infarto y $17,3 \%$ por revascularización) y de los fallecidos $(6,7 \%), 67 \%$ fue por causa cardiovascular.

\section{Discusión}

Los resultados de este estudio muestran que una alta proporción de pacientes está en tratamiento con los fármacos indicados en prevención secundaria después de implementada la cobertura universal de GES en infarto. Sin embargo, el logro de las metas terapéuticas para los factores de riesgo estudiados, principalmente aquellos que requieren cambios en los estilos de vida, como IMC y actividad física, es insuficiente. Esta es la primera evaluación del manejo ambulatorio del IAM con posterioridad a la implementación de las GES.

Nuestros resultados son similares a los reportados en estudios internacionales (EUROASPIRE, REACH, US NHANES 2005-2006, CHAMP), que muestran insuficiente control de los factores de riesgo cardiovascular en pacientes post-infarto ${ }^{34-37}$. En cuanto a la terapia, cabe señalar que el empleo de medicamentos en Chile fue superior a lo reportado recientemente en el Estudio PURE para países de similar nivel de desarrollo ${ }^{38}$.

En relación a los factores de riesgo modificables no hubo reducción del peso en promedio entre la hospitalización y el año, y una minoría de los pacientes realizaba actividad física en forma regular, coincidiendo con la baja participación de profesionales no médicos en la atención. La obesidad se asocia con otros factores de riesgo -presión arterial, lípidos sanguíneos y tolerancia a la glucosa ${ }^{39}$-, y la pérdida de peso ha mostrado impactar positivamente en su control y mejorar la calidad de vida ${ }^{40}$. Por otra parte, el ejercicio físico ayuda a mantener la baja de peso, tiene un efecto beneficioso en la circulación coronaria, la función endotelial y en los otros factores de riesgo ${ }^{12,24,41,42}$. El mayor logro se observó en la proporción de pacientes que dejaron de fumar post-infarto, sin embargo, este resultado debe ser interpretado con cautela, ya que el tabaquismo se evaluó por auroreporte, pudiendo existir una subestimación. Iestra et al reportaron un efecto cercano a 35\% en reducción de mortalidad en pacientes que dejan de fumar después de un infarto ${ }^{15}$.

Con la implementación de GES una alta pro- porción de pacientes se encontraba en tratamiento farmacológico. Pese a esto, el control de la presión arterial fue sub óptimo, lo que en otros estudios se ha relacionado con estilos de vida poco saludables $^{43-45}$; en nuestro estudio estos pacientes presentaron un IMC mayor. La presión arterial elevada en pacientes post-infarto se asocia con mayor riesgo de muerte y nuevos eventos coronarios ${ }^{46}$. En los pacientes diabéticos, sólo la mitad logró cifras de $\mathrm{Hb} 1 \mathrm{aC}<7 \%$; adicionalmente $45 \%$ de los pacientes no diabéticos presentaron glicemias en ayunas $>100 \mathrm{mg} / \mathrm{dl}$, indicando anormalidades del metabolismo de la glucosa en ellos. Ambas condiciones aumentan el riesgo en pacientes post-infarto ${ }^{47,48} \mathrm{lo}$ que sugiere la necesidad de evaluación y control estricto de los factores de riesgo en este grupo.

El acceso a fármacos encontrado en GES muestra adherencia a las guías clínicas y disponibilidad de éstos en los servicios de salud. Sobre $80 \%$ se encontraba en tratamiento con estatinas y más de $70 \%$ alcanzó LDL $<100 \mathrm{mg} / \mathrm{dl}$. Estos resultados son posiblemente producto que, en su diseño, la guía GES está enfocada principalmente al tratamiento farmacológico, con menor énfasis en la modificación de los factores de riesgo conductuales, viéndose reflejado en la baja participación de profesionales no médicos en la atención. Sin embargo, al considerar la actual recomendación en pacientes coronarios ( $\mathrm{LDL}<70 \mathrm{mg} / \mathrm{dl}$ ), sólo $30 \%$ alcanza esta cifra $^{49}$. El empleo de aspirina y betabloquedores -fármacos de reconocida eficacia en reducir la morbimortalidad post-infarto ${ }^{50}$ - fue cercano a $70 \%$ y $90 \%$, pero aún no se indican en todos los pacientes que no presentan contraindicaciones, especialmente en los de mayor edad.

Entre las principales limitaciones hay que mencionar el carácter no aleatorio de la muestra, con lo cual el estudio no representa a todos los pacientes con infarto atendidos en el sector público. Sin embargo, dada las características de los hospitales participantes -de referencia regional, similares a otros hospitales de igual complejidad del sector público-, y al carácter universal de la garantía, estos resultados podrían representar lo que ocurre en otros centros. En segundo lugar, el Registro GEMI no incluye todos los casos de IAM introduciendo un sesgo de selección al estudio; sin embargo, no se encontraron diferencias en cuanto a edad y sexo entre todos los egresos y la muestra incluida en GEMI. Finalmente, debido a las pérdidas de seguimiento, estos resultados pudiesen 
subestimar el logro de las metas, dado que estos eran pacientes más jóvenes. Como fortalezas destaca el seguimiento activo y el uso de mediciones estandarizadas en la mayoría de las variables.

En conclusión, la prevención secundaria postinfarto implementada en las garantías GES se caracteriza por adherir a las recomendaciones de las guías clínicas, insuficiente logro de las metas terapéuticas para los factores de riesgo conductuales, realización principalmente en el nivel secundario de atención por especialistas y baja participación de profesionales no médicos en el cuidado de los pacientes.

\section{Referencias}

1. Bases de defunciones 2010 [citado el 2 de noviembre de 2012]. Disponible en: http:www.deis.cl.

2. Departamento de Epidemiología. Estudio carga de enfermedad y carga atribuible 2007. 2008 [citado el 12 de noviembre de 2012]; Disponible en: http://epi.minsal.cl/ epi/html/invest/cargaenf2008/minuta21-07-2008.pdf.

3. Ministerio de Salud de Chile. Los Objetivos Sanitarios para la Década 2000-2010 Evaluación de final del período, Grado de cumplimiento de los objetivos de Impacto. 2010 [citado el 9 de diciembre de 2010]; Disponible en: http://epi.minsal.cl/epi/html/sdesalud/OS/Evaluacion ObjetivosSanitarios2000-2010.pdf.

4. Bhatt DL, Steg PG, Ohman EM, Hirsch AT, Ikeda Y, Mas JL, et al. International prevalence, recognition, and treatment of cardiovascular risk factors in outpatients with atherothrombosis. JAMA 2006; 295 (2): 180-9.

5. De Bacquer D, De Backer G, Ostor E, Simon J, Pyorala K. Predictive value of classical risk factors and their control in coronary patients: a follow-up of the EUROASPIRE I cohort. Eur J Cardiovasc Prev Rehabil 2003; 10 (4): 28995.

6. Hosokawa S, Hiasa Y, Miyazaki S, Ogura R, Miyajima H, Ohara $\mathrm{Y}$, et al. Effects of smoking cessation on coronary endothelial function in patients with recent myocardial infarction. Int J Cardiol 2008; 128 (1): 48-52.

7. Klein S, Burke LE, Bray GA, Blair S, Allison DB, PiSunyer X, et al. Clinical implications of obesity with specific focus on cardiovascular disease: a statement for professionals from the American Heart Association Council on Nutrition, Physical Activity, and Metabolism: endorsed by the American College of Cardiology Foundation. Circulation 2004; 110 (18): 2952-67.

8. Todd Miller M, Lavie CJ, White CJ. Impact of obesity on the pathogenesis and prognosis of coronary heart disease. J Cardiometab Syndr 2008; 3 (3): 162-7.
9. Sarwar N, Gao P, Seshasai SR, Gobin R, Kaptoge S, Di Angelantonio E, et al. Diabetes mellitus, fasting blood glucose concentration, and risk of vascular disease: a collaborative meta-analysis of 102 prospective studies. Lancet; 375 (9733): 2215-22.

10. Rosendorff C, Black HR, Cannon CP, Gersh BJ, Gore J, Izzo JL Jr, et al. Treatment of hypertension in the prevention and management of ischemic heart disease: a scientific statement from the American Heart Association Council for High Blood Pressure Research and the Councils on Clinical Cardiology and Epidemiology and Prevention. Circulation 2007; 115 (21): 2761-88.

11. Chow CK, Jolly S, Rao-Melacini P, Fox KA, Anand SS, Yusuf S. Association of diet, exercise, and smoking modification with risk of early cardiovascular events after acute coronary syndromes. Circulation 2010; 121 (6): 750-8.

12. Clark AM, Hartling L, Vandermeer B, McAlister FA. Meta-analysis: secondary prevention programs for patients with coronary artery disease. Ann Intern Med 2005; 143 (9): 659-72.

13. Critchley J, Capewell S. Smoking cessation for the secondary prevention of coronary heart disease. Cochrane Database Syst Rev 2004 (1): CD003041.

14. Gerber Y, Rosen LJ, Goldbourt U, Benyamini Y, Drory Y. Smoking status and long-term survival after first acute myocardial infarction a population-based cohort study. J Am Coll Cardiol 2009; 54 (25): 2382-7.

15. Iestra JA, Kromhout D, van der Schouw YT, Grobbee DE, Boshuizen HC, van Staveren WA. Effect size estimates of lifestyle and dietary changes on all-cause mortality in coronary artery disease patients: a systematic review. Circulation 2005; 112 (6): 924-34.

16. Bramlage P, Messer C, Bitterlich N, Pohlmann C, Cuneo A, Stammwitz E, et al. The effect of optimal medical therapy on 1-year mortality after acute myocardial infarction. Heart; 96 (8): 604-9.

17. Chew DP, Huynh L, Astley C, Liew D, Soman A, Brieger D. Potential Survival Gains in the Treatment of Myocardial Infarction. Heart 2009 Aug 6.

18. Freemantle N, Cleland J, Young P, Mason J, Harrison J. beta Blockade after myocardial infarction: systematic review and meta regression analysis. BMJ 1999; 318 (7200): 1730-7.

19. Van de Werf F, Bax J, Betriu A, Blomstrom-Lundqvist C, Crea F, Falk V, et al. Management of acute myocardial infarction in patients presenting with persistent STsegment elevation: the Task Force on the Management of ST-Segment Elevation Acute Myocardial Infarction of the European Society of Cardiology. Eur Heart J 2008; 29 (23): 2909-45. 
20. LaRosa JC, He J, Vupputuri S. Effect of statins on risk of coronary disease: a meta-analysis of randomized controlled trials. JAMA 1999; 282 (24): 2340-6.

21. Baigent C, Blackwell L, Emberson J, Holland LE, Reith C, Bhala N, et al. Efficacy and safety of more intensive lowering of LDL cholesterol: a meta-analysis of data from 170,000 participants in 26 randomised trials. Lancet 2010; 376 (9753): 1670-81.

22. Smith SC Jr, Allen J, Blair SN, Bonow RO, Brass LM, Fonarow GC, et al. AHA/ACC guidelines for secondary prevention for patients with coronary and other atherosclerotic vascular disease: 2006 update: endorsed by the National Heart, Lung, and Blood Institute. Circulation 2006 May 16; 113 (19): 2363-72.

23. Piepoli MF, Corra U, Benzer W, Bjarnason-Wehrens B, Dendale P, Gaita D, et al. Secondary prevention through cardiac rehabilitation: from knowledge to implementation. A position paper from the Cardiac Rehabilitation Section of the European Association of Cardiovascular Prevention and Rehabilitation. Eur J Cardiovasc Prev Rehabil 2010; 17 (1): 1-17.

24. Taylor RS, Brown A, Ebrahim S, Jolliffe J, Noorani H, Rees $\mathrm{K}$, et al. Exercise-based rehabilitation for patients with coronary heart disease: systematic review and meta-analysis of randomized controlled trials. Am J Med 2004; 116 (10): 682-92.

25. Dalal HM, Zawada A, Jolly K, Moxham T, Taylor RS. Home based versus centre based cardiac rehabilitation: Cochrane systematic review and meta-analysis. BMJ 2010; 340: b5631.

26. Ley 19.966: ESTABLECE UN REGIMEN DE GARANTIAS EN SALUD, (2005). Disponible en: http://www. redsalud.gov.cl/archivos/guiasges/GES_2007_final.pdf

27. Ministerio de Salud de Chile. Guía Clínica Infarto Agudo del Miocardio y Manejo del Dolor Torácico en Unidades de Emergencia 2005. 2005 [citado el 2 de noviembre de 2012]; Disponible en: http://www.redsalud.gov.cl/ archivos/saludcardiovascular/INFARTOAGUDO.pdf.

28. Ministerio de Salud Gobierno de Chile. Implementación del enfoque de riesgo en el Programa de Salud Cardiovascular. [citado el 9 de noviembre de 2012]; Disponible en: http://www.redsalud.gov.cl/portal/url/item/787e476 5248bc9e0e04001011f0172b5.pdf.

29. Nazzal NC, Campos TP, Corbalan HR, Lanas ZF, Bartolucci JJ, Sanhueza CP, et al. Impacto del plan AUGE en el tratamiento de pacientes con infarto agudo al miocardio con supradesnivel ST, en hospitales chilenos. Rev Med Chile 2008; 136 (10): 1231-9.

30. Thygesen K, Alpert JS, White HD, Jaffe AS, Apple FS, Galvani $M$, et al. Universal definition of myocardial infarction. Circulation 2007; 116 (22): 2634-53.
31. Kotseva K. Treatment of patients with coronary heart disease fails to meet standards of european guidelines: results of EUROASPIRE surveys. Rev Esp Cardiol 2009; 62 (10): 1095-8.

32. Ministerio de Salud Gobierno de Chile. Guía Clínica infarto agudo del miocardio con supradesnivel del segmento st. 2010; 2009 [citado el 13 de noviembre de 2012]; Disponible en: http://www.redsalud.gov.cl/portal/ url/item/72213ed52c3323d1e04001011f011398.pdf.

33. Ministerio de Salud de Chile. Guía Clínica 2009 Hipertensión Arterial Primaria o esencial en personas de 15 años y más. Febrero de 2010; [citado el 13 de noviembre de 2012]; Disponible en: http://www.redsalud.gov.cl/ portal/url/item/7220fdc4341c44a9e04001011f0113b9. pdf.

34. Vulic D, Lee BT, Dede J, López VA, Wong ND. Extent of Control of Cardiovascular Risk Factors and Adherence to Recommended Therapies in US Multiethnic Adults with Coronary Heart Disease: From a 2005-2006 National Survey. Am J Cardiovasc Drugs 2010; 10 (2): 109-14.

35. Kotseva K, Wood D, De Backer G, De Bacquer D, Pyorala $\mathrm{K}$, Keil U. Cardiovascular prevention guidelines in daily practice: a comparison of EUROASPIRE I, II, and III surveys in eight European countries. Lancet 2009; 373 (9667): 929-40.

36. Cacoub PP, Zeymer U, Limbourg T, Baumgartner I, Poldermans D, Rother J, et al. Effects of adherence to guidelines for the control of major cardiovascular risk factors on outcomes in the REduction of Atherothrombosis for Continued Health (REACH) Registry Europe. Heart 2011; 97 (8): 660-7.

37. Fonarow GC, Gawlinski A, Moughrabi S, Tillisch JH. Improved treatment of coronary heart disease by implementation of a Cardiac Hospitalization Atherosclerosis Management Program (CHAMP). Am J Cardiol 2001; 87 (7): 819-22.

38. Yusuf S, Islam S, Chow CK, Rangarajan S, Dagenais G, Díaz R, et al. Use of secondary prevention drugs for cardiovascular disease in the community in high-income, middle-income, and low-income countries (the PURE Study): a prospective epidemiological survey. Lancet 2011; 378 (9798): 1231-43.

39. Poirier P, Giles TD, Bray GA, Hong Y, Stern JS, Pi-Sunyer FX, et al. Obesity and cardiovascular disease: pathophysiology, evaluation, and effect of weight loss: an update of the 1997 American Heart Association Scientific Statement on Obesity and Heart Disease from the Obesity Committee of the Council on Nutrition, Physical Activity, and Metabolism. Circulation 2006; 113 (6): 898-918.

40. Lavie CJ, Milani RV, Artham SM, Patel DA, Ventura HO. The obesity paradox, weight loss, and coronary disease. 
Am J Med 2009; 122 (12): 1106-14.

41. Trzos E, Kurpesa M, Rechcinski T, Wierzbowska-Drabik K, Krzeminska-Pakula M. The influence of physical rehabilitation on arterial compliance in patients after myocardial infarction. Cardiol J 2007; 14 (4): 366-71.

42. Boraita A. Ejercicio, piedra angular de la prevención cardiovascular. Rev Esp Cardiol 2008; 61: 514-28.

43. Whelton SP, Chin A, Xin X, He J. Effect of aerobic exercise on blood pressure: a meta-analysis of randomized, controlled trials. Ann Intern Med 2002; 136 (7): 493 503.

44. Appel LJ, Frohlich ED, Hall JE, Pearson TA, Sacco RL, Seals DR, et al. The importance of populationwide sodium reduction as a means to prevent cardiovascular disease and stroke: a call to action from the American Heart Association. Circulation 2011; 123 (10): 1138-43.

45. Sacks FM, Svetkey LP, Vollmer WM, Appel LJ, Bray GA, Harsha D, et al. Effects on blood pressure of reduced dietary sodium and the Dietary Approaches to Stop Hypertension (DASH) diet. DASH-Sodium Collaborative Research Group. N Engl J Med 2001; 344 (1): 3-10.

46. Pedrinelli R, Ballo P, Fiorentini C, Denti S, Galderisi M, Ganau A, et al. Hypertension and acute myocardial infarction: an overview. J Cardiovasc Med (Hagerstown). 2012; 13 (3): 194-202.

47. Huxley R, Barzi F, Woodward M. Excess risk of fatal coronary heart disease associated with diabetes in men and women: meta-analysis of 37 prospective cohort studies. BMJ 2006; 332 (7533): 73-8.

48. Bartnik M, Ryden L, Malmberg K, Ohrvik J, Pyorala $\mathrm{K}$, Standl E, et al. Oral glucose tolerance test is needed for appropriate classification of glucose regulation in patients with coronary artery disease: a report from the Euro Heart Survey on Diabetes and the Heart. Heart 2007; 93 (1): 72-7.

49. Smith SC Jr, Benjamin EJ, Bonow RO, Braun LT, Creager MA, Franklin BA, et al. AHA/ACCF Secondary Prevention and Risk Reduction Therapy for Patients with Coronary and other Atherosclerotic Vascular Disease: 2011 update: a guideline from the American Heart Association and American College of Cardiology Foundation. Circulation 2011; 124 (22): 2458-73.

50. Van de Werf F, Bax J, Betriu A, Blomstrom-Lundqvist C, Crea F, Falk V, et al. ESC guidelines on management of acute myocardial infarction in patients presenting with persistent ST-segment elevation. Rev Esp Cardiol 2009; 62 (3): 293. 